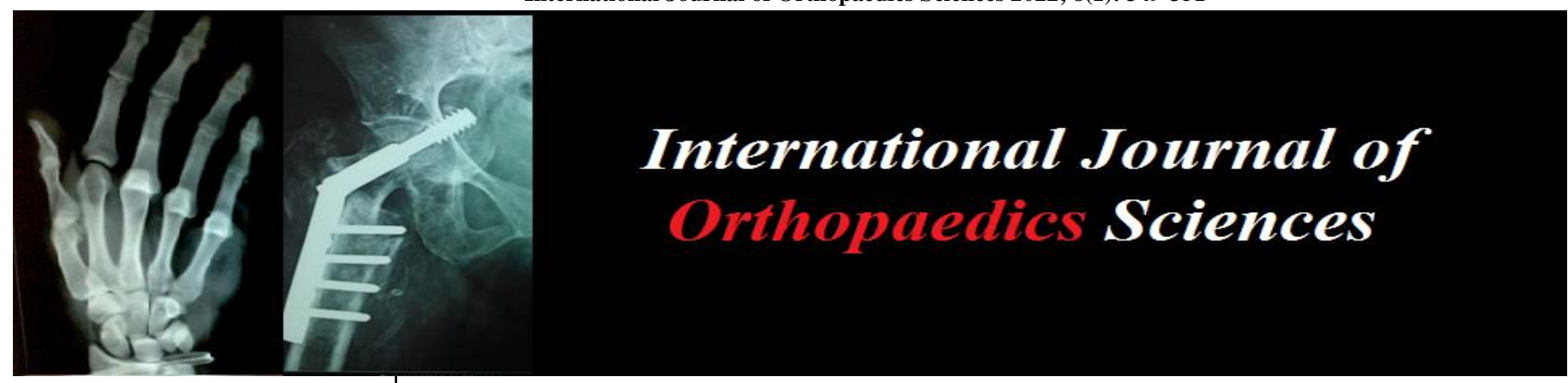

E-ISSN: 2395-1958

P-ISSN: 2706-6630

IJOS 2022; 8(1): 349-351

(C) 2022 IJOS

www.orthopaper.com

Received: 24-11-2021

Accepted: 26-12-2021

Dr. Neelanagowda VP Patil Professor and HOD, MMC \& RI,

Mysore, Karnataka, India

Dr. Arun GR

Assistant Professor, MMC \& RI,

Mysore, Karnataka, India

Dr. Deepak MK

Assistant Professor, MMC \& RI,

Mysore, Karnataka, India

Dr. Ravi GR

Junior Resident, MMC \& RI,

Mysore, Karnataka, India
Corresponding Author:

Dr. Ravi GR

Junior Resident, MMC \& RI,

Mysore, Karnataka, India

\section{Treating AC joint dislocation in pediatric population}

\author{
Dr. Neelanagowda VP Patil, Dr. Arun GR, Dr. Deepak MK and Dr. Ravi \\ GR
}

DOI: https://doi.org/10.22271/ortho.2022.v8.i1e.3041

Abstract

Acromioclavicular joint dislocation accounts for approximately $9 \%$ of shoulder girdle injuries. These injuries are classified into type I-VI injuries on the basis of the radiographic findings using the Rockwood criteria. Most surgical procedures involving the AC joint primarily involve fixation of the joint and reconstruction of the CC ligament. In children the Ac joint dislocations should not be treated with hook plates or any other rigid plate fixations. Operative intervention is recommended for complete acromioclavicular joint dislocation to restore AC stability, but the best operative technique is still controversial.

Patient and Methods: Young patients less than 14 yrs. are considered for this study with more than type 3 dislocation, here suture is used mostly ethibond to reconstruct the Ac joint dislocation .In this study patient were treated with figure of 8 suture reconstruction of Joint .

Results: Out of 10 patients 9 patients follow-up for 6 months were satisfactory reduction and maintenance of joint reduction, 1 patient got minimal displacement post-Surgery, No superficial infections found during the follow-up. Functional and radiological outcomes were good in 9 patients with mallet grade 5 and one case had grade 4 outcome.

Conclusion: Ac joint reconstruction with Ethibond has proved more suitable in both functional outcome along with no need of resurvey or removal.

Keywords: AC Joint, AC stability, CC ligaments

\section{Introduction}

About $70 \%$ to $80 \%$ of clavicle fractures occur in the middle part of the Clavicle. A combined injury involving a fracture of the lateral end of clavicle and dislocation of

the acromioclavicular joint is very uncommon. Dislocations of AC joints is a rare injuries in childhood. In children periarticular metaphyseal clavicle fractures many can lead to epiphyseal injury on fixation with plates or screws the treatment of AC Joint Injury has many controversies. The main dispute has been with the nonsurgical treatment versus surgical reconstruction for dislocations. There is a difficult in using metal implants for AC Joint Dislocation in Pediatric Patients due to thin skin and Epiphyseal Injury Chance. There was a need for a method with high Reduction and Tensile strength without injuring the Epiphysis or the Joint. Ethibond suture has a Polybraided fibres, good strength and easy to use. Though there was a good use with Ethibond overall in orthopaedics. There are minimal studies regarding the fixation reduction of $\mathrm{AC}$ joint in pediatric patients. In our study $\mathrm{AC}$ Joint Dislocation which was a less reported Injury in many pediatric Patients.

\section{Material and Methods}

This was a Prospective Study of 10 Patients with AC Joint Dislocation, followed for 6 months post operatively. All cases were closed type of Injury.8 patients were males and 2 were female patients in our study with age more than 8 yrs and less than 14 yrs. Many gives history of fall over the right shoulder. Here in our study we have operated with incision over the lateral end of clavicle extending to the acromion process with minimal extension to the deltoid fibers for adequate approach. All cases were operated within 7 days of admission so the dislocation was reducible easily without difficulty. After reduction we have confirmed with the help of the Carm. The ethibond of Number 5 can be used with a shape of Figure of 8 Manner, then it was tightened and reduction was achieved adequately with reinforcement of the ethibond ties. 
Wound was closed after wash with vicryl-2 0 with adequate bites of fascia and subcuticular tissue. Skin was closed with Ethilon 2-0 for good closure and healing

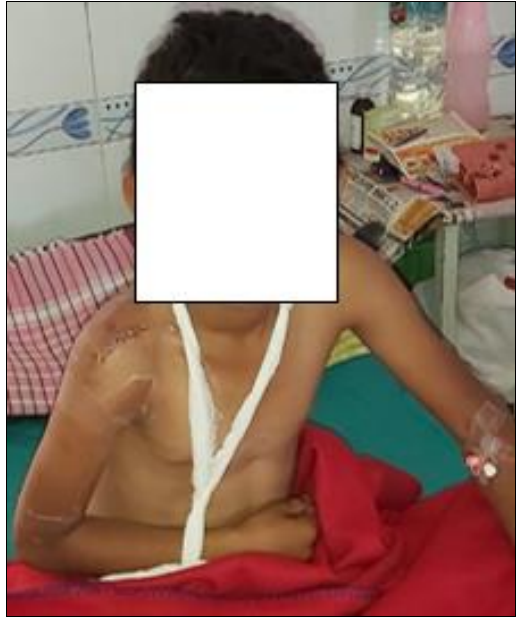

Fig 1: Post Operative Clinical Image

\section{Data Analysis}

Cases were followed up till 6 months of Postoperative period and the functional and radiological assessment was done regarding the outcome values. For Functional Outcome we have assessed with Mallet Scoring System and Radiologicaly assessed for union,reduction of joint dislocation.

The Mallet scoring system assessed grade of function was Grade -5 which is a normal shoulder.

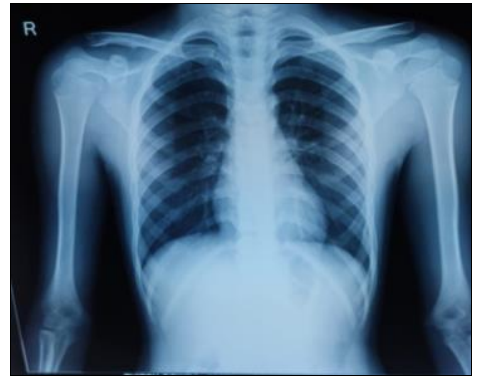

Fig 2: Final Follow-up X-Ray

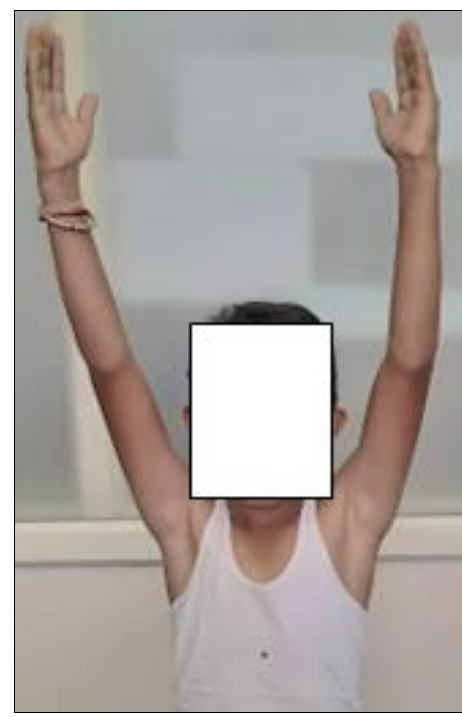

Fig 3: Final Clinical Follow-up

\section{Discussion}

At present, early AC joint fixations are followed for early rehabilitation and Functional Gain In many surgeons Now adays. There was a difficult in using metal high end implants as chance of epiphyseal injury with difficult skin care postsurgery. The use of a metal implant does not do justice in cases of AC Joint Dislocation type 2-3 with Clavicle Fractures so the need for implant with less chance of injury with less thickness but with good strength .The Ethibond was used in many cases with small fragment reconstruction in many adults and in patellar fractures .There can be a use of ethibond sutures in pediatric AC Joint reduction and fixation. But no studies have documented this adequately. The use of Hook Plate in AC Joint dislocation had many disadvantages like cut through, Arthritis, Epiphyseal fusion or injures. The use of K-Wire was considered in many studies with AC Dislocations in Paediatric patients. But the K-Wire was to pass from the epiphyseal Plate and Injury is high to epiphyseal plate. Also the K-Wire is less stable and need prolong stay in many cases and infection and discomfort is common with the use of K-wire use. Many Authors have documented some disadvantages with the use of K-wire for the $\mathrm{AC}$ joint reduction.

The use of Ethibond suture in the Figure of 8 Manner has given a good functional gain according to Mallet scoring System with good early healing of fracture. According to many other authors who have used Hook plate there was a functional gain of grade 4 in many cases with limitation in maximum abduction and external rotation due to impingement or itching to proximal humerus head.

In our study the kids resumed to daily activities within $4 \mathrm{wks}$ of the surgery and achieved maximum functional gain by 6 week .As compared to other studies with Plates or K-wires, the resumption of daily activities took about $6 \mathrm{wk}$ and maximum functional gain achieved by 10 weeks post operative days with adequate Physiotherapy help. In K- wire stabilization of AC Joint Dislocation there was some difficulty in achieving full range with K-wire .Even with adequate reduction the functional gain was Maximum achieved at 12 week post operative Period.

Thus there was a need for fixation without limiting the early mobilization, without injuring the epiphyseal plate and no need to remove the implant to minimize resurvey. Thus Ethibond in our case report has achieved all the needs as said above adequately in this type of a rare case study.

\section{Conclusion}

Ac joint reconstruction with Ethibond has proved more suitable in both functional outcome along with no need of resurvey or removal in this rare study. The Ethibond Use also have given early Rehabilitation chance in our study without any residual injury to the epiphyseal plate. Thus concluding the use of Ethibond in such cases in pediatric cases can be justified as good.

\section{References}

1. Asadollahi S, Bucknill A. Acute medial clavicle fracture in adults: a systematic review of demographics, clinical features and treatment outcomes in 220 patients. J Orthop Traumatol. 2019;20(1):24. https://doi.org/10.1186/s10195-019-0533-3 PMID: 31254115

2. Van Tongel A, McRae S, Gilhen A, Leiter J, MacDonald P. Management of anterior sternoclavicular dislocation: a survey of orthopaedic surgeons. Acta Orthop Belg. 2012;78(2):164-9. PMID: 22696984

3. Pediatric acromioclavicular and sternoclavicular joint dislocations PLOS ONE 
https://doi.org/10.1371/journal.pone.0244209 December 28, 202012 / 14

4. Maier D, Jaeger M, Izadpanah K, Bornebusch L, Sudkamp NP. [Traumatic injuries of the sternoclavicular joint]. Unfallchirurg. 2011;114(7):611-21. quiz 22-3. https://doi.org/10.1007/s00113-011-2049-z PMID: 21739356

5. Eidman DK, Siff SJ, Tullos HS. Acromioclavicular lesions in children. Am J Sports Med. 1981;9(3):150. https://doi.org/10.1177/036354658100900304 PMID: 7235110

6. Black GB, McPherson JA, Reed MH. Traumatic pseudodislocation of the acromioclavicular joint in children A fifteen year review. Am J Sports Med. 1991;19(6):644-6.

https://doi.org/10.1177/036354659101900616 PMID: 1781505

7. Galanis N, Anastasiadis P, Grigoropoulou F, Kirkos J, Kapetanos G. Judo-related traumatic posterior sternoclavicular joint dislocation in a child. Clin J Sport Med. 2014;24(3):271-3. https://doi.org/10.1097/JSM.0000000000000020 PMID: 24172655

8. Ngom G, Mohamed AS, El Housseine MO, Ndour O. Traumatic posterior sternoclavicular joint dislocation in a child: a case report. Pan Afr Med J. 2014;19:386. https://doi.org/10.11604/pamj.2014.19.386.5684 PMID: 25995782. 